\title{
The Effect of Bio-Fertilizers on Plant Growth and Growth Rate of Grafted Avocado (Persea americana Mill.)
}

\author{
Lazarus Agus Sukamto ${ }^{\#}$, Reni Lestari ${ }^{* 1}$, and Winda Utami Putri ${ }^{* 2}$

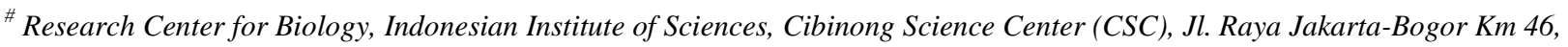 \\ Cibinong 16911, Bogor, West Java, Indonesia \\ E-mail: lazarus.agus@gmail.com
}

*Center for Plant Conservation-Botanic Gardens, Indonesian Institute of Sciences, Jl. Ir. H. Djuanda 13, Bogor 16003, West Java, Indonesia

Email: ${ }^{1}$ reni_naa@yahoo.com, ${ }^{2}$ winda.utami.putri@gmail.com

\begin{abstract}
Avocado (Persea americana Mill.) is considered the most nutritious of all fruits. Avocado fruit contain high unsaturated fat, protein, and energy. It could be eaten fresh for food, drinks, cooking, and cosmetics. Recently, it has become a significant commodity in international trade. Indonesia is the 2 nd avocado producing country, but only little quantity of avocado fruits could be exported. The farmers usually grow avocado plants from the seeds, without proper fertilizers in their backyards or small gardens. The problems could be solved by using grafted plants, proper fertilizers, and growing in a large scale of areas. This research was conducted to find out the effect of two liquid bio-fertilizers namely Mega Rhizo and Beyonic StarTmik on the plant growth and growth rate of grafted avocado plants. Some plant growths and growth rates of grafted avocado were influenced significantly by genotype accession, kind of bio-fertilizer, and weather (temperature). Plant growth and growth rate of most avocado accessions were not significant differences to bio-fertilizer applications, but some avocado accessions on certain months were significant differently. Growth rate ranks of plant height based on accession were no. 10, 28, 13, 1, 5, 2, and 14 consecutively. Those of canopy width were no. $28,10,1,2,14,5$, and 13 consecutively. Those of trunk diameters were no. 28, 10, 2, 5, 1, 13, and 14 consecutively. All growth rate ranks based on bio-fertilizer were Mega Rhizo, Beyonic StarTmik, and control consecutively.
\end{abstract}

Keywords — Grafted avocado; bio-fertilizer; Mega Rhizo; Beyonic StarTmik; plant growth; growth rate.

\section{INTRODUCTION}

Avocado belongs to Lauraceae family, originated from lowland and highland of meso-America, which cover West India, Guatemala, and Mexico regions. The native habitat of West Indian avocado is lowland, hot, and humid Central American forests with a short dry season; Guatemalan avocado is a tropical highland with year-round cool conditions; Mexican avocado is the most elevated (1400 $2500 \mathrm{~m}$ ), temperatures $14.2-19.80 \mathrm{C}$, rainfall $665-1562$ $\mathrm{mm}$, and $6-8$ months winter-spring dry period. Recognition of nutritional value of avocado fruit caused colonizers of the America to distribute this crop to new climate permitted regions [1].

FAOSTAT Database stated that avocado production increased rapidly in the world, the avocado was introduced to Indonesia by1750 [1]. It was brought into Indonesia by Spanish merchants, cultivated using seedlings in home gardens of mountain regions [4]. Indonesia produced avocado 294,200 metric tons, which was the 2nd in the world after 1st Mexico (1,316,104 metric tons) in 2012, but Indonesia did not recorded as avocado exporter country because of very small volume [5].

Avocado growth and production require 13 essential elements. These elements are divided three groups based on the relative amount required by avocado plant. The primary nutrients required are nitrogen, phosphorus, and potassium. The secondary nutrients required are calcium, magnesium and sulfur. The micro-nutrients required are zinc, iron, manganese, copper, boron, molybdenum, and chlorine [6]. Avocado plants require basic fertilizers, such as manure, inorganic fertilizers (urea, TSP, $\mathrm{KCl}$ ), also micro-nutrients. Application of in-organic fertilizer could cause a pollution of soil, water, expensive cost, also do not have micro-nutrient; whereas application of bio-fertilizer could increase activated respiration and enzymatic of plant roots, supply nutrients and plant hormones for plant growth [7], [8], [9], [10], also substitute some amount of in-organic and decrease production cost [11], [12].

Our experiment used Mega Rhizo and Beyonic StarTmik liquid bio-fertilizers. Mega Rhizo fertilizer contains 200 g/l 
C organic, $0.25 \mathrm{~g} / \mathrm{l} \mathrm{N}$ total, $0.1 \mathrm{~g} / \mathrm{l} \mathrm{P} 2 \mathrm{O} 5,1 \mathrm{~g} / \mathrm{l} \mathrm{K} 2 \mathrm{O}, 0.1 \mathrm{~g} / \mathrm{l}$ $\mathrm{Ca}, 52 \mathrm{mg} / \mathrm{l} \mathrm{Fe}, 0.27 \mathrm{mg} / \mathrm{l} \mathrm{Mn}, 0.33 \mathrm{mg} / \mathrm{l} \mathrm{Cu}, 0.15 \mathrm{mg} / \mathrm{l} \mathrm{Zn}$, and $1.97 \mathrm{mg} / \mathrm{l} \mathrm{B}$, also micro-organisms of Aspergillus niger, Lactobacillus sp., Actinomycetes sp., Streptomyces, and Acetobacter [13]. Beyonic StarTmik bio-fertilizer contains 106 - 107 number of micro-organisms (Bacillus, Pseudomonas, Painibacillus, and Burkholderia), dissolved phosphate, tied up with nitrogen, hormones producer (IAA, cytokinin, gibberelin), and bio-control, also improve soil's health, boost the plant's growth, increase plant production [14], [15]. The aims of this experiment were to know the affect of bio-fertilizers to plant growth and grow rate of seven accessions of grafted avocado plants in the field.

\section{METHODOLOGY}

There are 630 of grafted avocado plants consisted of seven accessions $(1,2,5,10,13,14,28)$ grown in Cibinong Science Center, Bogor since October 2011. Cibinong Science Center where located experiment is low elevated ( $207 \mathrm{~m}$ ), temperatures 24.6 - 26.40C, month rainfall 201 $702 \mathrm{~mm}$ with $19-87$ days rain, air humidity $78-89 \%$, and light intensity $201-356 \mathrm{cal} / \mathrm{cm} 2$ from January 2013 until April 2014 [2]. The analysis of soil before treatment were texture consisted of $83 \%$ lime, $15 \%$ dust, and $2 \%$ sand; soil $\mathrm{pH}$ (H2O) low (5), Ca 7.97 (very low), Mg 0.97 (low), K 0.04 (very low), Na 0.06 (very low), and KTK 16.37 (low) [3].

Basic fertilizers of $10 \mathrm{~kg}$ manure and $100 \mathrm{~g}$ NPK $(15: 15: 15)$ were applied on soil where grafted avocado would be planted. The basic fertilizers of $10 \mathrm{~kg}$ manure and $200 \mathrm{~g}$ NPK were applied on each plant every six months. The experiment was carried out from October 2012 until April 2014. The experiment was arranged in randomized complete design. The treatments consisted of three application (two fertilizers and control) and seven accessions. Each accession consisted of three replications and each replication consisted of seven plants. The plant number of this experiment was 147 plants. Twelve month-old plants were applied with/ without two kinds of liquid bio-fertilizer, namely Mega Rhizo, Beyonic StarTmik, and control. Mega Rhizo fertilizer was applied every three months as recommended dosage, namely 120 times dilution and 3 liter for each plant for 4 times. Beyonic StarTmik fertilizer was applied every month as recommended dosage, namely 100 times dilution and 0.5 liter for each plant for 12 times.

Observation was conducted every two months, started on 5 th month until 17 th month after bio-fertilizers application in October 2012. The parameters of observation covered measuring plant growth (plant height, canopy width, trunk diameter) and growth rate (rank based on accession and fertilizer). Plant height was measured from soil surface to highest shoot, canopy width was measured from the longest canopy plant, and trunk diameter was measure from soil surface to $20 \mathrm{~cm}$ of the plant trunk. Growth rate was measured from plant growth of the month was subtracted by previous month and divided by previous month. Rank of growth rate based on accession was measured from average of growth rate of each accession, whereas based on fertilizer was measured from average of growth rate of fertilizer application. Data was analyzed with ANOVA procedures of
SPSS, means comparison was continued analyzed with Duncan's Multiple Range Test based on $\alpha=5 \%$.

\section{RESULTS AND DISCUSSION}

Grafted avocado plants treated with bio-fertilizers showed differently on plant growth and growth rate of each accession. Most application of bio-fertilizers did not affect significantly on plant growth and growth rate of avocado plants, only a few of the accessions could increase their growth and grow rate on certain months of observation. Soil analysis showed most nutrients content of the soil increased after eleven months bio-fertilizer application [16].

\section{A. Plant height}

1) Plant Growth: The growth of plant height treated with liquid fertilizers showed differently each accession. Plant height accession no. 2 was significant different on 7th, 9th, and 11th months. The highest plant height was treated by Mega Rhizo fertilizer $(199.85 \mathrm{~cm})$, followed by Beyonic StarTmik $(165.50 \mathrm{~cm})$ and control $(164.00 \mathrm{~cm})$ on 7 th , until $247.29 \mathrm{~cm}, 212.43 \mathrm{~cm}$, and $199.71 \mathrm{~cm}$ on 11 th month consecutively (Table I). Plant height accession no. 13 was significant different since on 9th until 17th months. The highest plant height was treated by Mega Rhizo fertilizer $(183.43 \mathrm{~cm})$, followed by Beyonic StarTmik $(168.86 \mathrm{~cm})$ and control (143.67) on 9th month until 269.71, 222.57, $205.83 \mathrm{~cm}$ on 17 th month consecutively (Table I). Plant heights of other accessions (no. 1, 5, 10, 14, 28) were not significantly different among fertilizer treatments and control (Table I).

These results stated that genotype of avocado plants affected their responses to the bio-fertilizers. Avocado accession no. 13 was the most responses to application of Mega Rhizo fertilizer, which showed the highest plant height compared to those of Beyonic StarTmik and control on 9th to 17 th months. Avocado accession no. 2 also had same responses to the Mega Rhizo and Beyonic StarTmik biofertilizers but only on 7th to 11th months. Cultivars and rootstocks of avocado influenced nutrients absorption for plant growth [17]. Beyonic StarTmik also increased plant height of water melon (Citrullus lanatus) [10]. Mega Rhizo or Beyonic StarTmik increased plant growth, yield, and decreased applying in-organic fertilizer on maize [11].

2) Growth Rate: different on 7th month. The highest growth rate was treated by Mega Rhizo fertilizer $(0.123 \mathrm{~cm})$, followed by control $(0.060 \mathrm{~cm})$ and Beyonic StarTmik $(0.055 \mathrm{~cm})$ (Table II). Growth rate accession no. 17 was significant different on 7 th month. The highest growth rate was treated Beyonic StarTmik fertilizer $(0.089 \mathrm{~cm})$, followed by Mega Rhizo $(0.080 \mathrm{~cm})$ and control $(0.066 \mathrm{~cm})$ consecutively (Table II). Growth rate accession no. 1 was significant different on 5th month. The highest growth rate was treated by control $(0.183 \mathrm{~cm})$, followed by Mega Rhizo fertilizer $(0.120 \mathrm{~cm})$, and Beyonic StarTmik $(0.094 \mathrm{~cm})$ consecutively (Table II). These results showed that weather and plant genotype affected on growth rate of plant height. Avocado accession no. 1 responded to plant genotype, which control showed the highest growth rate on 5th month, whereas accession no. 2 were the highest growth rate with application of Mega Rhizo fertilizer on 7th month. On the 
other hand, avocado accession no. 14 was the highest growth rate with application of Beyonic StarTmik fertilizer on 7 th month. The rank (from the highest to the slowest) of growth rate of plant height based on accession was no. 10, 28, 13, 1, 5,2 , and 14 consecutively (Table III), whereas those of based on fertilizer application was Mega Rhizo, Beyonic StarTmik, and control consecutively (Table III).

\section{B. Canopy width}

\section{1) Plant Growth:}

The growth of canopy width showed did not significantly different treated with liquid fertilizers for all accessions, except of accession no. 13. Canopy growth of accession no. 13 showed differently on 11th until 17th month. The canopy widest was treated with Mega Rhizo fertilizer $(155.29 \mathrm{~cm})$, followed by Beyonic StarTmik fertilizer $(145.86 \mathrm{~cm})$, and control $(120 \mathrm{~cm})$ consecutively on 11th month. The canopy widest was treated (189.71), followed by Beyonic StarTmik $(172.86 \mathrm{~cm})$ and control $(147.67 \mathrm{~cm})$ consecutively on 17 th month (Table IV). There was a different rank of canopy width only of the accession plants no 13 started on 11th month. It could be caused by different genotype of plant that responsed differently to the bio-fertilizer applied.

2) Growth Rate: The growth rate of canopy width showed significantly different treated with liquid fertilizers on three accessions but occurring sporadic. Avocado accession no. 14 showed significantly different on 17 th month. The fastest growth rate of canopy width was treated Beyonic StarTmik $(0.071 \mathrm{~cm})$, followed by Mega Rhizo fertilizers $(0.064 \mathrm{~cm})$ and control $(0.007 \mathrm{~cm})$ (Table V). Avocado accession no. 13 showed significantly different on 7 th and 15th months. The fastest growth rate of canopy width was Mega Rhizo fertilizer $(0.130 \mathrm{~cm})$, followed by control $(0.080 \mathrm{~cm})$ and Beyonic StarTmik fertilizer $(0.076$ $\mathrm{cm})$ on 7 th month; whereas on 15 th month, the fastest of growth rate was control $(0.065 \mathrm{~cm})$, followed by Mega Rhizo fertilizer $(0.051 \mathrm{~cm})$ and Beyonic StarTmik fertilizer $(0.047 \mathrm{~cm})$ (Table V). Avocado accession no. 10 showed significantly different on 17 th month. The fastest on growth rate was Mega Rhizo fertilizer $(0.087 \mathrm{~cm})$, followed by control $(0.073 \mathrm{~cm})$ and Beyonic StarTmik fertilizer $(0.060$ $\mathrm{cm}$ ) (Table V). The rank (from the highest to the slowest) of growth rate of canopy width were accession no. 28, 10, 1, 2, 14,5 , and 13 consecutively (VI), whereas those of based on fertilizer application was Mega Rhizo, Beyonic StarTmik, and control consecutively (Table VI). Mega Rhizo was better than Beyonic StarTmik fertilizer because Mega Rhizo content micronutrients of $\mathrm{Fe}$ and $\mathrm{Zn}$ that required highly by avocado plant [13], [18].

\section{Trunk Diameter}

1) Plant Growth: The growth of trunk diameter were not significant different, except accession no. 13. The significant differently of trunk diameter growth began on 9th until 17th months. The widest trunk growth was Mega Rhizo fertilizer (44.29 mm), followed by Beyonic StarTmik (40.64 $\mathrm{mm})$ and control $(34.00 \mathrm{~mm})$ on 9 th month consecutively until $54.71,50.42,41.50$ on 17 th month consecutively (Table VII). These results showed that only plant genotype accession no. 13 was the most response to the bio-fertilizer.
Mega Rhizo fertilizer treatment was more response than Beyonic StarTmik for trunk diameter of avocado plant.

2) Growth Rate: The growth rate of trunk diameter showed significantly different on 7 th month of accession no. 10,13 , and 14. The fastest of growth rate of accession no. 10 was Beyonic StarTmik $(0.161 \mathrm{~mm})$, followed by control $(0.128 \mathrm{~mm})$ and Mega Rhizo fertilizers $(0.089 \mathrm{~mm})$ consecutively (Table VIII). The fastest of growth rate of accession no. 13 was Beyonic StarTmik $(0.146 \mathrm{~mm})$, followed by Mega Rhizo fertilizers $(0.134 \mathrm{~mm})$ and control $(0.078 \mathrm{~mm})$ consecutively (Table VIII). The fastest of growth rate of accession no. 14 was Mega Rhizo fertilizer $(0.160 \mathrm{~mm})$, followed by Beyonic StarTmik $(0.097 \mathrm{~mm})$ and control $(0.051 \mathrm{~mm})$ consecutively (Table VIII). These results showed the genotype of plant avocado affected primarily to growth rate of trunk diameter. The analysis of soil sample showed an application of Mega Rhizo fertilizer also increased significantly nitrogen content $(0.17 \%$ to $0.24 \%$ ) in soil, which could increase growth rate of trunk diameter. The weather also affected to growth rate of trunk diameter accession no. 14, 13, and 10 that significantly differences occurred on 7 th month. The temperatures of 7th month (May 2013) and one month before (April 2013) were the highest $(26.2$ - 26.40C) compared to other months on experimental location. Climatic conditions influenced nutrients absorption for growth rate of avocado [17]. The rank (from the highest to the slowest) of growth rate of trunk diameter was accession no. 28, 10, 2, 5, 1, 13, and 14 (Table IX), whereas those of based on fertilizer application was Mega Rhizo, Beyonic StarTmik, and control consecutively (Table IX).

\section{CONCLUSIONS}

Application of bio-fertilizers affected differently on plant growth and growth rate of grafted avocado. Plant growth and growth rate of grafted avocado were influenced by genotype accession, kind of bio-fertilizer, and weather (temperature). Plant growth of most avocado accessions was not significant differences to bio-fertilizer application, but avocado accession no. 13 was significant different on 9th until 17th months. Some growth rate of avocado accession no. 14, 13, and 10 affected significantly on 7th month when high temperature occurred. The fastest growth rate based on accession was avocado accession no. 28, whereas based on bio-fertilizer was Mega Rhizo.

\section{ACKNOWLEDGMENT}

We are thankful to research competitive board of Indonesian Institute of Sciences (LIPI) for funding this research, also thanks to Gozali Sumaatmadja, Dodi Sutardi, Majen, and Madin for research helps.

\section{REFERENCES}

[1] R. J. Knight Jr., History, Distribution and Uses. A. W. Whiley, B. Schaffer, and B. N. Wolstenholme, Eds. The avocado, Botany, Production and Uses. Oxon, UK. CABI Publishing, 2002.

[2] "Badan Meteorologi Klimatologi dan Geofisika," Data Iklim, Stasiun Klimatologi Darmaga Bogor, Indonesia, August 19, 2014.

[3] "Laboratorium Tanah," Hasil Analisis Contoh Tanah, Bogor, Indonesia, Nov. 5, 2012. 
[4] S. S. Harjadi. Avocado Production in Indonesia. M. K. Papademetriou, Ed. Avocado Production in Asia and the Pacific. Bangkok, Thailand, July 2000.[Online]. Available: http://www.fao.org/docrep/003/x6902e/x6902e07.htm. Accessed on August 26, 2014.

[5] Avocado Statistics, 2001-2012 World Avocado Production. [Online]. Available: http://www.novagrim.com/Pages/2000_ 2011_avocado_ statistics_EN.aspx. Accessed on August 26, 2014.

[6] G. S. Bender. (1999) Avocado Fertilization. Chapter 2, Book 2 California Cooperative Extension. San Diego, CA. [Online] Available:

http://scholar.googleusercontent.com/scholar?q=cache:r3jfqEXAMZ gJ:scholar.google.com/\&hl=id\&as_sdt=0,5. Accessed on August 15, 2014.

[7] S. Antonius, D. Agustyani, M. Rahmansyah, and B. Martono. "Development of sustainable agriculture: soil microorganisms enzymatic activity of organic farming in Jabopuncur catchment's area treated with agricultural wastes as biofertilizer" In Proc. Int. Sem. Fac. Biology, UGM, Yogyakarta. 2007. pp. 340-341, 2007.

[8] S. Antonius, D. Agustyani, M. Rahmansyah, and B. Martono. Eksplorasi dan penapisan mikroba dari Malinau sebagai agen hayati pendukung pertanian yang berkelanjutan. Proseding Lingkungan Hidup. Seminar Nasional Biologi XX dan Kongres PBI XIV. UIN Maliki, Malang, pp. 347-357. 2009.

[9] M. Ramansyah, S. Antonius, and N. Sulistinah. Phosphatase and urease instability caused by pesticides present in soil improved by grounded rice straw. ARPN Journal of Agricultural and Biological Science. Vol. 4(2), pp. 56-62, 2009.
[10] S. Antonius and D. Agustiyani. " Pengaruh pupuk organic hayati yang mengandung mikroba bermanfaat terhadap pertumbuhan dan hasil panen tanaman semangka serta sifat biokimia tanahnya pada percobaan lapangan di Malinau-Kalimantan Timur". Berkala Penelitian Hayati. Vol. 16, pp. 203-206. 2011.

[11] T. Juhaeti, N. Hidayati, and M. Rahmansyah. "Pertumbuhan dan produksi jagung pulut lokal Sulawesi Selatan yang ditanam di polibag pada berbagai kombinasi perlakuan pupuk organic", Jurnal Biologi Indonesia, vol. 9(2), pp. 219-232, 2013.

[12] M. Ramansyah, N. Hidayati, T. Juhaeti, and A. Sugiharto. "Effect of bio-inorganic fertilizer on productivity improvement of well adapted local maize (Zea mays ceratina L.) variety", ARPN Journal of Agricultural and Biological Science, vol. 8 (3), pp. 233-240, 2013.

[13] Pupuk Organik Cair Mega Rhizo. PT THS Govarindo Lestari, Klaten, Indonesia.

[14] Pupuk Organik Hayati Beyonic StarTmik. Pusat Penelitian BiologiLIPI, Cibinong, Indonesia.

[15] D. Nugraha and Ani H. (2013) Bioorganic fertilizer LIPI style. [Online]. Available: http://en.voi.co.id/voi-features/5281-bioorganicfertilizer-lipi-style. Accessed on August 7, 2014.

[16] "Laboratorium Tanah," Hasil Analisis Contoh Tanah, Bogor, Indonesia, Sep. 23, 2013.

[17] E. Lahav and A. W. Whiley, Irrigation and Mineral Nutrition. A. W. Whiley, B. Schaffer, and B. N. Wolstenholme, Eds. The avocado, Botany, Production and Uses. Oxon, UK. CABI Publishing, 2002.

[18] K. Joyce. (2014) Liquid fertilizers for avocado. [Online]. Available: http://www.ehow.com.info_12209044_liquid-fertilizersavocados.html, Accessed on july 25, 2014. 


\section{APPENDIX}

TABLE I

Plant Height GROWTH (CM) OF GRAFTED AVOCADO FOR 17 MONTHS

\begin{tabular}{|c|c|c|c|c|c|c|c|c|}
\hline \multirow{2}{*}{ Acc. } & \multirow{2}{*}{ Treatment } & \multicolumn{7}{|c|}{ Months After Bio-fertilizer Application } \\
\hline & & 5 & 7 & 9 & 11 & 13 & 15 & 17 \\
\hline \multirow{3}{*}{1} & Control & $303.57 \pm 82.60(a)$ & $333.57 \pm 91.88(a)$ & $365.14 \pm 95.52(a)$ & $431.71 \pm 136.86(a)$ & $464.00 \pm 133.92(a)$ & $480.14 \pm 136.48(a)$ & $495.14 \pm 134.24(a)$ \\
\hline & Megarhizo & $271.71 \pm 49.57(a)$ & $306.86 \pm 40.15(a)$ & $341.14 \pm 53.23(a)$ & $393.29 \pm 62.89$ (a) & $418.43 \pm 66.22(a)$ & $447.29 \pm 63.52(a)$ & $465.00 \pm 67.76(a)$ \\
\hline & Beyonik & $287.00 \pm 90.01(\mathrm{a})$ & $329.60 \pm 109.44(a)$ & $354.33 \pm 103.02(\mathrm{a})$ & $412.83 \pm 139.98(\mathrm{a})$ & $438.00 \pm 144.58(a)$ & $450.17 \pm 144.97(\mathrm{a})$ & $470.50 \pm 140.34(a)$ \\
\hline \multirow{3}{*}{2} & Control & $154.57 \pm 31.44(\mathrm{a})$ & $164.00 \pm 30.60(a)$ & $175.43 \pm 32.40(a)$ & $199.71 \pm 36.84(a)$ & $212.71 \pm 43.25(a)$ & $224.14 \pm 44.24(a)$ & $237.71 \pm 53.20(a)$ \\
\hline & Megarhizo & $175.43 \pm 30.02(a)$ & $199.85 \pm 31.90(b)$ & $216.43 \pm 38.74(b)$ & $247.29 \pm 36.48(b)$ & $258.00 \pm 37.61(a)$ & $260.14 \pm 38.16(a)$ & $283.29 \pm 39.22(\mathrm{a})$ \\
\hline & Beyonik & $155.83 \pm 12.01(\mathrm{a})$ & $165.50 \pm 18.38(a)$ & $191.57 \pm 24.15(\mathrm{ab})$ & $212.43 \pm 25.47(\mathrm{ab})$ & $224.29 \pm 41.71(\mathrm{a})$ & $247.00 \pm 48.21(\mathrm{a})$ & $267.00 \pm 48.63(\mathrm{a})$ \\
\hline \multirow{3}{*}{5} & Control & $152.43 \pm 34.65(a)$ & $167.29 \pm 40.68(a)$ & $182.14 \pm 48.36(\mathrm{a})$ & $203.43 \pm 55.61(\mathrm{a})$ & $224.86 \pm 51.09(\mathrm{a})$ & $236.00 \pm 50.48(a)$ & $253.57 \pm 52.70(\mathrm{a})$ \\
\hline & Megarhizo & $178.29 \pm 40.49$ (a) & $192.57 \pm 45.00(\mathrm{a})$ & $209.29 \pm 54.74(a)$ & $246.57 \pm 62.33(\mathrm{a})$ & $252.86 \pm 67.71(a)$ & $266.14 \pm 67.57(a)$ & $281.29 \pm 68.30$ (a) \\
\hline & Beyonik & $156.00 \pm 38.52(a)$ & $168.00 \pm 51.59(a)$ & $193.14 \pm 68.64(a)$ & $208.86 \pm 66.02(a)$ & $228.14 \pm 77.55(a)$ & $241.71 \pm 77.08(a)$ & $260.00 \pm 78.00(a)$ \\
\hline \multirow{3}{*}{10} & Control & $116.33 \pm 18.37$ (a) & $130.17 \pm 19.31(a)$ & $131.33 \pm 19.99$ (a) & $145.83 \pm 23.75$ (a) & $177.50 \pm 25.74(a)$ & $191.50 \pm 26.46(\mathrm{a})$ & $217.83 \pm 27.29$ (a) \\
\hline & Megarhizo & $123.86 \pm 12.81(\mathrm{a})$ & $136.00 \pm 14.83(\mathrm{a})$ & $147.14 \pm 31.78(a)$ & $160.86 \pm 32.87(a)$ & $185.00 \pm 43.65(\mathrm{a})$ & $198.14 \pm 43.88(\mathrm{a})$ & $222.57 \pm 50.62(a)$ \\
\hline & Beyonik & $118.43 \pm 17.32(a)$ & $129.71 \pm 20.93(a)$ & $138.00 \pm 27.47(a)$ & $154.14 \pm 33.21(\mathrm{a})$ & $189.00 \pm 27.36(\mathrm{a})$ & $203.29 \pm 27.37$ (a) & $223.71 \pm 29.66(a)$ \\
\hline \multirow{3}{*}{13} & Control & $118.67 \pm 29.95(a)$ & $134.17 \pm 26.90(a)$ & $143.67 \pm 26.01(a)$ & $159.17 \pm 34.58(a)$ & $177.17 \pm 38.74(a)$ & $190.33 \pm 38.96$ (a) & $205.83 \pm 40.09$ (a) \\
\hline & Megarhizo & $142.14 \pm 31.86(\mathrm{ab})$ & $162.00 \pm 31.98(a)$ & $183.43 \pm 28.79(b)$ & $205.00 \pm 31.06(\mathrm{~b})$ & $226.14 \pm 35.42(b)$ & $240.57 \pm 35.64(b)$ & $269.71 \pm 62.21(b)$ \\
\hline & Beyonik & $150.57 \pm 17.79(\mathrm{~b})$ & $158.43 \pm 17.35$ (a) & $168.86 \pm 19.20(\mathrm{ab})$ & $179.86 \pm 17.62(\mathrm{ab})$ & $192.86 \pm 26.53(\mathrm{ab})$ & $207.00 \pm 26.75(\mathrm{ab})$ & $222.57 \pm 28.23(\mathrm{ab})$ \\
\hline \multirow{3}{*}{14} & Control & $161.57 \pm 24.31(\mathrm{a})$ & $173.00 \pm 23.72(a)$ & $191.57 \pm 25.39$ (a) & $205.71 \pm 32.38(a)$ & $229.71 \pm 40.93(a)$ & $241.86 \pm 41.41(a)$ & $246.86 \pm 40.11$ (a) \\
\hline & Megarhizo & $157.83 \pm 26.22(a)$ & $171.83 \pm 31.15(\mathrm{a})$ & $184.71 \pm 37.49$ (a) & $205.57 \pm 42.59$ (a) & $230.86 \pm 40.37(a)$ & $251.57 \pm 56.74(a)$ & $258.14 \pm 39.30$ (a) \\
\hline & Beyonik & $159.86 \pm 26.51(a)$ & $176.57 \pm 35.31(a)$ & $195.43 \pm 45.92(a)$ & $215.71 \pm 46.51(a)$ & $238.85 \pm 51.51(a)$ & $244.29 \pm 49.00(a)$ & $256.29 \pm 49.84(\mathrm{a})$ \\
\hline \multirow{3}{*}{28} & Control & $97.43 \pm 20.72(a)$ & $113.71 \pm 25.36(a)$ & $123.86 \pm 25.31(a)$ & $139.71 \pm 32.59$ (a) & $147.86 \pm 30.84(a)$ & $157.71 \pm 31.26(\mathrm{a})$ & $172.86 \pm 30.71(a)$ \\
\hline & Megarhizo & $100.29 \pm 20.30(a)$ & $115.29 \pm 18.25(a)$ & $128.14 \pm 23.36(a)$ & $143.57 \pm 25.10(a)$ & $152.57 \pm 28.91(a)$ & $163.29 \pm 30.21(a)$ & $179.14 \pm 29.94(a)$ \\
\hline & Beyonik & $94.00 \pm 13.81(\mathrm{a})$ & $108.57 \pm 17.02(a)$ & $118.29 \pm 16.79$ (a) & $136.00 \pm 22.82(\mathrm{a})$ & $143.00 \pm 25.14(\mathrm{a})$ & $154.14 \pm 23.08(a)$ & $168.71 \pm 23.12(a)$ \\
\hline
\end{tabular}

Values represents means \pm SE. Different letters in the same parameter data indicate significant differences by DUNCAN test (p $\leq 0.05$ ) 
TABLE II

Growth RATE (CM/MONTH) OF Plant Height OF GRAFTED AVOCADO

\begin{tabular}{|c|c|c|c|c|c|c|c|c|}
\hline \multirow{2}{*}{ Acc. } & \multirow{2}{*}{ Treatment } & \multicolumn{7}{|c|}{ Months After Bio-fertilizer Application } \\
\hline & & 5 & 7 & 9 & 11 & 13 & 15 & 17 \\
\hline \multirow{3}{*}{1} & Control & $0.183 \pm 0.070(\mathrm{~b})$ & $0.086 \pm 0.034(\mathrm{a})$ & $0.090 \pm 0.044(a)$ & $0.139 \pm 0.085(a)$ & $0.074 \pm 0.046(\mathrm{a})$ & $0.036 \pm 0.011(\mathrm{a})$ & $0.033 \pm 0.024(\mathrm{a})$ \\
\hline & Megarhizo & $0.120 \pm 0.057(\mathrm{ab})$ & $0.119 \pm 0.068(a)$ & $0.094 \pm 0.046$ (a) & $0.131 \pm 0.049$ (a) & $0.057 \pm 0.072(\mathrm{a})$ & $0.066 \pm 0.078(a)$ & $0.039 \pm 0.009$ (a) \\
\hline & Beyonik & $0.094 \pm 0.052(a)$ & $0.122 \pm 0.041(a)$ & $0.238 \pm 0.376(a)$ & $0.130 \pm 0.048(a)$ & $0.057 \pm 0.038(\mathrm{a})$ & $0.028 \pm 0.008(a)$ & $0.048 \pm 0.033(\mathrm{a})$ \\
\hline \multirow{3}{*}{2} & Control & $0.087 \pm 0.061(\mathrm{a})$ & $0.060 \pm 0.024(\mathrm{a})$ & $0.063 \pm 0.046(a)$ & $0.117 \pm 0.079(a)$ & $0.057 \pm 0.053(\mathrm{a})$ & $0.050 \pm 0.016(\mathrm{a})$ & $0.054 \pm 0.026(\mathrm{a})$ \\
\hline & Megarhizo & $0.103 \pm 0.063(\mathrm{a})$ & $0.123 \pm 0.046(b)$ & $0.073 \pm 0.052(\mathrm{a})$ & $0.126 \pm 0.072(a)$ & $0.040 \pm 0.052(\mathrm{a})$ & $0.001 \pm 0.133(\mathrm{a})$ & $0.083 \pm 0.036(\mathrm{a})$ \\
\hline & Beyonik & $0.130 \pm 0.057$ (a) & $0.055 \pm 0.055$ (a) & $0.259 \pm 0.329$ (a) & $0.094 \pm 0.072$ (a) & $0.041 \pm 0.087(\mathrm{a})$ & $0.087 \pm 0.090$ (a) & $0.076 \pm 0.036$ (a) \\
\hline \multirow{3}{*}{5} & Control & $0.129 \pm 0.072$ (a) & $0.084 \pm 0.034(a)$ & $0.074 \pm 0.054(a)$ & $0.101 \pm 0.059$ (a) & $0.104 \pm 0.063(\mathrm{a})$ & $0.050 \pm 0.020$ (a) & $0.069 \pm 0.011$ (a) \\
\hline & Megarhizo & $0.147 \pm 0.053$ (a) & $0.073 \pm 0.032(a)$ & $0.067 \pm 0.067$ (a) & $0.139 \pm 0.129$ (a) & $0.004 \pm 0.247(\mathrm{a})$ & $0.056 \pm 0.021$ (a) & $0.059 \pm 0.022(\mathrm{a})$ \\
\hline & Beyonik & $0.130 \pm 0.065(a)$ & $0.051 \pm 0.926(a)$ & $0.113 \pm 0.088$ (a) & $0.087 \pm 0.063$ (a) & $0.074 \pm 0.056(\mathrm{a})$ & $0.064 \pm 0.029$ (a) & $0.077 \pm 0.021$ (a) \\
\hline \multirow{3}{*}{10} & Control & $0.188 \pm 0.122(a)$ & $0.105 \pm 0.072(a)$ & $0.003 \pm 0.123(a)$ & $0.097 \pm 0.052(a)$ & $0.178 \pm 0.074(\mathrm{a})$ & $0.075 \pm 0.014(a)$ & $0.118 \pm 0.081(\mathrm{a})$ \\
\hline & Megarhizo & $0.151 \pm 0.091(a)$ & $0.087 \pm 0.046$ (a) & $0.050 \pm 0.154(a)$ & $0.089 \pm 0.081(a)$ & $0.120 \pm 0.080(a)$ & $0.071 \pm 0.015(\mathrm{a})$ & $0.103 \pm 0.084(\mathrm{a})$ \\
\hline & Beyonik & $0.157 \pm 0.139$ (a) & $0.086 \pm 0.042(a)$ & $0.053 \pm 0.071(\mathrm{a})$ & $0.101 \pm 0.057(\mathrm{a})$ & $0.187 \pm 0.082(\mathrm{a})$ & $0.071 \pm 0.012(\mathrm{a})$ & $0.093 \pm 0.019$ (a) \\
\hline \multirow{3}{*}{13} & Control & $0.080 \pm 0.116$ (a) & $0.115 \pm 0.086$ (a) & $0.067 \pm 0.042(a)$ & $0.092 \pm 0.040$ (a) & $0.102 \pm 0.055(\mathrm{a})$ & $0.070 \pm 0.015$ (a) & $0.077 \pm 0.012$ (a) \\
\hline & Megarhizo & $0.169 \pm 0.065(\mathrm{a})$ & $0.116 \pm 0.143(a)$ & $0.117 \pm 0.102(a)$ & $0.103 \pm 0.064(a)$ & $0.091 \pm 0.062(\mathrm{a})$ & $0.061 \pm 0.011(\mathrm{a})$ & $0.093 \pm 0.097(\mathrm{a})$ \\
\hline & Beyonik & $0.127 \pm 0.059$ (a) & $0.051 \pm 0.029$ (a) & $0.061 \pm 0.011(\mathrm{a})$ & $0.061 \pm 0.036(a)$ & $0.061 \pm 0.052(a)$ & $0.070 \pm 0.008(a)$ & $0.070 \pm 0.008(a)$ \\
\hline \multirow{3}{*}{14} & Control & $0.103 \pm 0.064(a)$ & $0.066 \pm 0.033$ (a) & $0.099 \pm 0.032(a)$ & $0.066 \pm 0.024(a)$ & $0.097 \pm 0.080(\mathrm{a})$ & $0.049 \pm 0.013$ (a) & $0.019 \pm 0.083$ (a) \\
\hline & Megarhizo & $0.163 \pm 0.034(\mathrm{a})$ & $0.080 \pm 0.049(\mathrm{~b})$ & $0.213 \pm 0.351(a)$ & $0.101 \pm 0.034(a)$ & $0.110 \pm 0.053(\mathrm{a})$ & $0.074 \pm 0.062(a)$ & $0.034 \pm 0.079$ (a) \\
\hline & Beyonik & $0.106 \pm 0.061$ (a) & $0.089 \pm 0.060(\mathrm{ab})$ & $0.090 \pm 0.047$ (a) & $0.094 \pm 0.046$ (a) & $0.071 \pm 0.258(\mathrm{a})$ & $0.009 \pm 0.181(\mathrm{a})$ & $0.047 \pm 0.026(\mathrm{a})$ \\
\hline \multirow{3}{*}{28} & Control & $0.184 \pm 0.098(a)$ & $0.139 \pm 0.046(a)$ & $0.083 \pm 0.061(a)$ & $0.109 \pm 0.065(a)$ & $0.059 \pm 0.053(\mathrm{a})$ & $0.064 \pm 0.019$ (a) & $0.089 \pm 0.020$ (a) \\
\hline & Megarhizo & $0.147 \pm 0.109$ (a) & $0.131 \pm 0.100(a)$ & $0.099 \pm 0.046(a)$ & $0.054 \pm 0.087(a)$ & $0.054 \pm 0.040(\mathrm{a})$ & $0.067 \pm 0.021(\mathrm{a})$ & $0.090 \pm 0.017(\mathrm{a})$ \\
\hline & Beyonik & $0.177 \pm 0.072$ (a) & $0.131 \pm 0.039$ (a) & $0.081 \pm 0.062(a)$ & $0.049 \pm 0.083(a)$ & $0.049 \pm 0.031(\mathrm{a})$ & $0.076 \pm 0.033(a)$ & $0.089 \pm 0.028(a)$ \\
\hline
\end{tabular}

Values represents means \pm SE. Different letters in the same parameter data indicate significant differences by DUNCAN test (p $\leq 0.05$ )

TABLE III

Growth Rate (CM/MONTH) OF Plant Height BASED ON BIO-FERTILIZER AND ACCESSION NuMBER

\begin{tabular}{|l|c|c|c|c|c|c|c|c|c|}
\hline \multirow{2}{*}{ Treatment } & \multicolumn{9}{|c|}{ Accession No. } \\
\cline { 2 - 10 } & 1 & 2 & 5 & 10 & 13 & 14 & 28 & Average & Rank \\
\hline Control & 0.106 & 0.079 & 0.097 & 0.130 & 0.094 & 0.080 & 0.117 & 0.100 & 3 \\
\hline Megarhizo & 0.100 & 0.089 & 0.094 & 0.113 & 0.128 & 0.101 & 0.115 & 0.106 & 1 \\
\hline Beyonic & 0.091 & 0.108 & 0.099 & 0.122 & 0.079 & 0.088 & 0.118 & 0.101 & 2 \\
\hline Average & 0.099 & 0.092 & 0.097 & 0.121 & 0.100 & 0.090 & 0.117 & & \\
\hline Rank & 4 & 6 & 5 & 1 & 3 & 7 & 2 & & \\
\hline
\end{tabular}


TABLE IV

CANOPY WIDTH GROWTH (CM) OF GRAFTED AVOCADO FOR 17 MONTHS

\begin{tabular}{|c|c|c|c|c|c|c|c|c|}
\hline \multirow{2}{*}{ Acc. } & \multirow{2}{*}{ Treatment } & \multicolumn{7}{|c|}{ Months After Bio-fertilizer Application } \\
\hline & & 5 & 7 & 9 & 11 & 13 & 15 & 17 \\
\hline \multirow{3}{*}{1} & Control & $207.43 \pm 65.43(a)$ & $236.29 \pm 69.73(a)$ & $272.00 \pm 88.87(\mathrm{a})$ & $338.00 \pm 129.84(a)$ & $362.86 \pm 128.28(a)$ & $383.57 \pm 106.20(a)$ & $395.86 \pm 105.27(a)$ \\
\hline & Megarhizo & $183.86 \pm 26.53(\mathrm{a})$ & $205.71 \pm 32.14(a)$ & $232.86 \pm 39.07$ (a) & $278.57 \pm 45.89$ (a) & $310.43 \pm 54.95(a)$ & $346.00 \pm 66.57$ (a) & $361.29 \pm 67.18(a)$ \\
\hline & Beyonik & $201.17 \pm 40.32(a)$ & $232.83 \pm 62.00(a)$ & $265.17 \pm 87.91(a)$ & $306.67 \pm 96.11(a)$ & $327.00 \pm 90.30(a)$ & $344.83 \pm 73.29$ (a) & $361.83 \pm 69.78(\mathrm{a})$ \\
\hline \multirow{3}{*}{2} & Control & $112.57 \pm 19.75(a)$ & $127.14 \pm 32.89(a)$ & $140.00 \pm 29.92(a)$ & $152.43 \pm 31.04(\mathrm{a})$ & $165.43 \pm 43.23(\mathrm{a})$ & $175.86 \pm 43.31(\mathrm{a})$ & $185.14 \pm 46.24(a)$ \\
\hline & Megarhizo & $113.00 \pm 16.24(a)$ & $126.29 \pm 27.61(a)$ & $144.86 \pm 25.10(a)$ & $159.00 \pm 28.45(a)$ & $169.43 \pm 32.89$ (a) & $177.29 \pm 32.99$ (a) & $200.00 \pm 37.53(\mathrm{a})$ \\
\hline & Beyonik & $118.43 \pm 22.50(a)$ & $127.29 \pm 19.70(a)$ & $141.57 \pm 15.03(\mathrm{a})$ & $151.86 \pm 28.63(\mathrm{a})$ & $165.86 \pm 30.85$ (a) & $173.71 \pm 32.67(a)$ & $194.14 \pm 33.00(a)$ \\
\hline \multirow{3}{*}{5} & Control & $158.29 \pm 26.29$ (a) & $178.43 \pm 33.30(a)$ & $181.00 \pm 34.34(\mathrm{a})$ & $201.14 \pm 40.36(a)$ & $219.71 \pm 43.91(a)$ & $230.57 \pm 45.21(\mathrm{a})$ & $245.43 \pm 44.17(\mathrm{a})$ \\
\hline & Megarhizo & $185.29 \pm 28.87$ (a) & $208.43 \pm 36.85$ (a) & $227.14 \pm 45.60(a)$ & $245.57 \pm 49.66(a)$ & $269.00 \pm 43.28(\mathrm{a})$ & $277.14 \pm 43.98(a)$ & $294.29 \pm 43.27(\mathrm{a})$ \\
\hline & Beyonik & $156.43 \pm 47.40(a)$ & $177.57 \pm 60.89$ (a) & $187.43 \pm 68.95(\mathrm{a})$ & $213.00 \pm 76.77(\mathrm{a})$ & $233.57 \pm 78.81(a)$ & $240.43 \pm 80.02(a)$ & $257.86 \pm 83.97(a)$ \\
\hline \multirow{3}{*}{10} & Control & $103.00 \pm 11.01(\mathrm{a})$ & $125.00 \pm 19.37(\mathrm{a})$ & $135.17 \pm 18.32(a)$ & $146.17 \pm 14.68(a)$ & $185.17 \pm 30.33(\mathrm{a})$ & $193.83 \pm 29.55(\mathrm{a})$ & $209.50 \pm 33.76(\mathrm{a})$ \\
\hline & Megarhizo & $111.14 \pm 19.00(\mathrm{a})$ & $131.43 \pm 20.16(\mathrm{a})$ & $141.43 \pm 21.59(\mathrm{a})$ & $156.00 \pm 29.97(a)$ & $192.00 \pm 28.80$ (a) & $201.71 \pm 29.76(a)$ & $220.29 \pm 29.54(a)$ \\
\hline & Beyonik & $116.14 \pm 21.63(a)$ & $131.29 \pm 22.46(a)$ & $144.71 \pm 26.76(a)$ & $158.14 \pm 37.08(a)$ & $185.57 \pm 36.77$ (a) & $192.71 \pm 40.26(\mathrm{a})$ & $205.14 \pm 44.15(a)$ \\
\hline \multirow{3}{*}{13} & Control & $106.29 \pm 17.23(\mathrm{a})$ & $112.17 \pm 20.32(\mathrm{a})$ & $117.17 \pm 20.23(\mathrm{a})$ & $120.00 \pm 25.59$ (a) & $129.17 \pm 27.31(\mathrm{a})$ & $138.17 \pm 28.07(a)$ & $147.67 \pm 29.64(\mathrm{a})$ \\
\hline & Megarhizo & $114.00 \pm 17.37(\mathrm{a})$ & $131.43 \pm 19.36(a)$ & $140.43 \pm 16.11(\mathrm{a})$ & $155.29 \pm 21.45(\mathrm{~b})$ & $167.71 \pm 32.03(\mathrm{~b})$ & $176.71 \pm 32.08(b)$ & $189.71 \pm 31.61(b)$ \\
\hline & Beyonik & $123.43 \pm 20.95(a)$ & $133.14 \pm 20.29$ (a) & $140.71 \pm 19.70(\mathrm{a})$ & $145.86 \pm 21.03(\mathrm{ab})$ & $159.57 \pm 21.33(\mathrm{ab})$ & $168.00 \pm 23.68(\mathrm{ab})$ & $172.86 \pm 33.85(\mathrm{ab})$ \\
\hline \multirow{3}{*}{14} & Control & $139.00 \pm 18.78(a)$ & $149.57 \pm 16.02(a)$ & $164.14 \pm 15.16(\mathrm{a})$ & $186.71 \pm 15.13(\mathrm{a})$ & $205.14 \pm 24.59$ (a) & $214.71 \pm 26.58(a)$ & $218.71 \pm 39.72(a)$ \\
\hline & Megarhizo & $132.43 \pm 21.24(\mathrm{a})$ & $142.86 \pm 19.81(\mathrm{a})$ & $157.86 \pm 23.70$ (a) & $177.86 \pm 25.18(\mathrm{a})$ & $193.00 \pm 28.14(\mathrm{a})$ & $203.71 \pm 30.16$ (a) & $218.00 \pm 34.43(\mathrm{a})$ \\
\hline & Beyonik & $134.14 \pm 27.85(a)$ & $149.43 \pm 29.17(a)$ & $174.00 \pm 48.95$ (a) & $189.86 \pm 52.95(a)$ & $205.71 \pm 52.24(\mathrm{a})$ & $215.29 \pm 53.32(\mathrm{a})$ & $232.57 \pm 60.34(a)$ \\
\hline \multirow{3}{*}{28} & Control & $68.14 \pm 11.16(a)$ & $87.28 \pm 13.11(\mathrm{a})$ & $98.71 \pm 15.64(a)$ & $110.86 \pm 16.44(a)$ & $117.14 \pm 17.05(a)$ & $125.86 \pm 17.27$ (a) & $139.57 \pm 17.87(a)$ \\
\hline & Megarhizo & $66.57 \pm 13.62(a)$ & $86.86 \pm 15.87(a)$ & $94.29 \pm 20.20(a)$ & $110.57 \pm 12.15$ (a) & $118.57 \pm 9.45$ (a) & $128.43 \pm 9.95(a)$ & $140.43 \pm 7.91(a)$ \\
\hline & Beyonik & $63.71 \pm 20.74(a)$ & $82.57 \pm 16.37(a)$ & $98.14 \pm 19.17(a)$ & $110.43 \pm 13.99$ (a) & $112.71 \pm 12.98(a)$ & $120.57 \pm 12.55$ (a) & $133.14 \pm 10.27(a)$ \\
\hline
\end{tabular}

Values represents means \pm SE. Different letters in the same parameter data indicate significant differences by DUNCAN test (p $\leq 0.05)$ 
TABLE V

GROWTH RATE (CM/MONTH) OF CANOPY WIDTH OF GRAFTED AVOCADO

\begin{tabular}{|c|c|c|c|c|c|c|c|c|}
\hline \multirow{2}{*}{ Acc. } & \multirow{2}{*}{ Treatment } & \multicolumn{7}{|c|}{ Months After Bio-fertilizer Application } \\
\hline & & 5 & 7 & 9 & 11 & 13 & 15 & 17 \\
\hline \multirow{3}{*}{1} & Control & $0.174 \pm 0.083(\mathrm{a})$ & $0.123 \pm 0.107(\mathrm{a})$ & $0.119 \pm 0.067(\mathrm{a})$ & $0.164 \pm 0.120$ (a) & $0.081 \pm 0.060(\mathrm{a})$ & $0.077 \pm 0.138(a)$ & $0.034 \pm 0.017(\mathrm{a})$ \\
\hline & Megarhizo & $0.163 \pm 0.110(a)$ & $0.101 \pm 0.067(\mathrm{a})$ & $0.114 \pm 0.054(\mathrm{a})$ & $0.160 \pm 0.078(a)$ & $0.096 \pm 0.089(a)$ & $0.097 \pm 0.093(\mathrm{a})$ & $0.043 \pm 0.008(a)$ \\
\hline & Beyonik & $0.088 \pm 0.044(\mathrm{a})$ & $0.190 \pm 0.136(\mathrm{a})$ & $0.108 \pm 0.080(\mathrm{a})$ & $0.128 \pm 0.097(a)$ & $0.067 \pm 0.051(\mathrm{a})$ & $0.058 \pm 0.124(\mathrm{a})$ & $0.050 \pm 0.022(\mathrm{a})$ \\
\hline \multirow{3}{*}{2} & Control & $0.094 \pm 0.116(\mathrm{a})$ & $0.103 \pm 0.073(\mathrm{a})$ & $0.096 \pm 0.090(\mathrm{a})$ & $0.080 \pm 0.060(\mathrm{a})$ & $0.069 \pm 0.051(\mathrm{a})$ & $0.061 \pm 0.012(\mathrm{a})$ & $0.049 \pm 0.050(\mathrm{a})$ \\
\hline & Megarhizo & $0.124 \pm 0.092(\mathrm{a})$ & $0.091 \pm 0.107(a)$ & $0.131 \pm 0.076(\mathrm{a})$ & $0.083 \pm 0.098(\mathrm{a})$ & $0.060 \pm 0.036(\mathrm{a})$ & $0.044 \pm 0.013(\mathrm{a})$ & $0.110 \pm 0.079$ (a) \\
\hline & Beyonik & $0.103 \pm 0.062(a)$ & $0.073 \pm 0.063(\mathrm{a})$ & $0.104 \pm 0.069$ (a) & $0.056 \pm 0.089$ (a) & $0.084 \pm 0.072(\mathrm{a})$ & $0.044 \pm 0.019(\mathrm{a})$ & $0.103 \pm 0.098(a)$ \\
\hline \multirow{3}{*}{5} & Control & $0.090 \pm 0.061(\mathrm{a})$ & $0.107 \pm 0.068(\mathrm{a})$ & $0.013 \pm 0.011(\mathrm{a})$ & $0.099 \pm 0.034(\mathrm{a})$ & $0.083 \pm 0.031(\mathrm{a})$ & $0.050 \pm 0.006(b)$ & $0.066 \pm 0.017(\mathrm{a})$ \\
\hline & Megarhizo & $0.060 \pm 0.063(\mathrm{a})$ & $0.106 \pm 0.085(\mathrm{a})$ & $0.074 \pm 0.078(\mathrm{a})$ & $0.071 \pm 0.067$ (a) & $0.091 \pm 0.081(\mathrm{a})$ & $0.030 \pm 0.014(\mathrm{a})$ & $0.059 \pm 0.038(\mathrm{a})$ \\
\hline & Beyonik & $0.101 \pm 0.081(\mathrm{a})$ & $0.104 \pm 0.081(\mathrm{a})$ & $0.036 \pm 0.106(a)$ & $0.123 \pm 0.037(a)$ & $0.091 \pm 0.089$ (a) & $0.031 \pm 0.016(\mathrm{a})$ & $0.071 \pm 0.034(\mathrm{a})$ \\
\hline \multirow{3}{*}{10} & Control & $0.177 \pm 0.069$ (a) & $0.170 \pm 0.098(\mathrm{a})$ & $0.075 \pm 0.060(\mathrm{a})$ & $0.078 \pm 0.052(\mathrm{a})$ & $0.202 \pm 0.077(\mathrm{a})$ & $0.045 \pm 0.014(\mathrm{a})$ & $0.073 \pm 0.024(\mathrm{ab})$ \\
\hline & Megarhizo & $0.199 \pm 0.079$ (a) & $0.154 \pm 0.077$ (a) & $0.067 \pm 0.062$ (a) & $0.089 \pm 0.063(\mathrm{a})$ & $0.187 \pm 0.085(\mathrm{a})$ & $0.050 \pm 0.012(\mathrm{a})$ & $0.087 \pm 0.020(\mathrm{~b})$ \\
\hline & Beyonik & $0.231 \pm 0.120$ (a) & $0.117 \pm 0.050(\mathrm{~b})$ & $0.090 \pm 0.061(\mathrm{a})$ & $0.079 \pm 0.061(\mathrm{a})$ & $0.147 \pm 0.084(\mathrm{a})$ & $0.036 \pm 0.018(\mathrm{a})$ & $0.060 \pm 0.012(\mathrm{a})$ \\
\hline \multirow{3}{*}{13} & Control & $0.136 \pm 0.148(\mathrm{a})$ & $0.080 \pm 0.049(\mathrm{ab})$ & $0.045 \pm 0.031(\mathrm{a})$ & $0.017 \pm 0.042(\mathrm{a})$ & $0.070 \pm 0.086(\mathrm{a})$ & $0.065 \pm 0.019(\mathrm{~b})$ & $0.062 \pm 0.021(\mathrm{a})$ \\
\hline & Megarhizo & $0.126 \pm 0.073(\mathrm{a})$ & $0.130 \pm 0.048(b)$ & $0.063 \pm 0.110$ (a) & $0.091 \pm 0.062(\mathrm{a})$ & $0.067 \pm 0.090(\mathrm{a})$ & $0.051 \pm 0.015(\mathrm{ab})$ & $0.070 \pm 0.024(\mathrm{a})$ \\
\hline & Beyonik & $0.161 \pm 0.086(\mathrm{a})$ & $0.076 \pm 0.031(\mathrm{a})$ & $0.056 \pm 0.052$ (a) & $0.294 \pm 0.085(\mathrm{~b})$ & $0.089 \pm 0.050(\mathrm{a})$ & $0.047 \pm 0.010(\mathrm{a})$ & $0.016 \pm 0.119$ (a) \\
\hline \multirow{3}{*}{14} & Control & $0.081 \pm 0.091(\mathrm{a})$ & $0.071 \pm 0.051(\mathrm{a})$ & $0.087 \pm 0.075(\mathrm{a})$ & $0.119 \pm 0.063(\mathrm{a})$ & $0.084 \pm 0.084(\mathrm{a})$ & $0.044 \pm 0.011(\mathrm{a})$ & $0.007 \pm 0.080(\mathrm{a})$ \\
\hline & Megarhizo & $0.106 \pm 0.060(\mathrm{a})$ & $0.074 \pm 0.035(\mathrm{~b})$ & $0.091 \pm 0.050$ (a) & $0.109 \pm 0.071(\mathrm{a})$ & $0.073 \pm 0.098(\mathrm{a})$ & $0.053 \pm 0.018(\mathrm{a})$ & $0.064 \pm 0.020(\mathrm{ab})$ \\
\hline & Beyonik & $0.066 \pm 0.052(\mathrm{a})$ & $0.103 \pm 0.041(\mathrm{a})$ & $0.121 \pm 0.100(\mathrm{a})$ & $0.081 \pm 0.057(\mathrm{a})$ & $0.083 \pm 0.035(\mathrm{a})$ & $0.046 \pm 0.014(\mathrm{a})$ & $0.071 \pm 0.041(\mathrm{~b})$ \\
\hline \multirow{3}{*}{28} & Control & $0.136 \pm 0.109$ (a) & $0.216 \pm 0.106(a)$ & $0.111 \pm 0.084(\mathrm{a})$ & $0.104 \pm 0.108(a)$ & $0.053 \pm 0.057(\mathrm{a})$ & $0.070 \pm 0.024(\mathrm{a})$ & $0.100 \pm 0.028(\mathrm{a})$ \\
\hline & Megarhizo & $0.210 \pm 0.120(\mathrm{a})$ & $0.229 \pm 0.098(\mathrm{a})$ & $0.070 \pm 0.104(\mathrm{a})$ & $0.147 \pm 0.157$ (a) & $0.070 \pm 0.055$ (a) & $0.077 \pm 0.031$ (a) & $0.086 \pm 0.036$ (a) \\
\hline & Beyonik & $0.090 \pm 0.367(a)$ & $0.239 \pm 0.129$ (a) & $0.146 \pm 0.155(a)$ & $0.119 \pm 0.085(\mathrm{a})$ & $0.019 \pm 0.076(\mathrm{a})$ & $0.066 \pm 0.025(\mathrm{a})$ & $0.096 \pm 0.033(\mathrm{a})$ \\
\hline
\end{tabular}

Values represents means \pm SE. Different letters in the same parameter data indicate significant differences by DUNCAN test ( $\leq 0$.05)

TABLE VI

GROWTH RATE (CM/MONTH) OF CANOPY WIDTH BASED ON BIO-FERTILIZER AND ACCESSION NUMBER

\begin{tabular}{|l|c|c|c|c|c|c|c|c|c|}
\hline \multirow{2}{*}{ Treatment } & \multicolumn{9}{|c|}{ Accession No. } \\
\cline { 2 - 11 } & 1 & 2 & 5 & 10 & 13 & 14 & 28 & Average & Rank \\
\hline Control & 0.132 & 0.09 & 0.081 & 0.133 & 0.07 & 0.082 & 0.135 & 0.103 & 3 \\
\hline Megarhizo & 0.13 & 0.106 & 0.078 & 0.14 & 0.097 & 0.092 & 0.157 & 0.114 & 1 \\
\hline Beyonic & 0.113 & 0.091 & 0.093 & 0.129 & 0.078 & 0.094 & 0.14 & 0.105 & 2 \\
\hline Average & 0.125 & 0.095 & 0.084 & 0.134 & 0.082 & 0.89 & 0.144 & & \\
\hline Rank & 3 & 4 & 6 & 2 & 7 & 5 & 1 & & \\
\hline
\end{tabular}


TABLE VII

TrunK DiAmETER Growth (MM) OF GRAFTED AVOCADO FOR 17 MONTHS

\begin{tabular}{|c|c|c|c|c|c|c|c|c|}
\hline \multirow{2}{*}{ Acc } & \multirow{2}{*}{ Treatment } & \multicolumn{7}{|c|}{ Months After Bio-fertilizer Application } \\
\hline & & 5 & 7 & 9 & 11 & 13 & 15 & 17 \\
\hline \multirow{3}{*}{1} & Control & $47.71 \pm 12.08(\mathrm{a})$ & $57.00 \pm 7.62(a)$ & $62.71 \pm 11.24(\mathrm{a})$ & $74.57 \pm 17.27(\mathrm{a})$ & $79.29 \pm 19.59$ (a) & $79.29 \pm 19.59(a)$ & $80.29 \pm 19.77(a)$ \\
\hline & Megarhizo & $47.86 \pm 5.27(a)$ & $56.29 \pm 6.99(a)$ & $63.14 \pm 8.93(a)$ & $69.43 \pm 10.52(a)$ & $75.71 \pm 13.61(a)$ & $75.71 \pm 13.61(a)$ & $77.43 \pm 13.02(a)$ \\
\hline & Beyonik & $51.00 \pm 11.47(\mathrm{a})$ & $58.00 \pm 10.75(a)$ & $67.33 \pm 13.52(a)$ & $78.17 \pm 17.24(\mathrm{a})$ & $82.67 \pm 18.35$ (a) & $83.00 \pm 18.09$ (a) & $84.00 \pm 18.09(\mathrm{a})$ \\
\hline \multirow{3}{*}{2} & Control & $31.86 \pm 5.05(a)$ & $35.14 \pm 4.91(a)$ & $39.13 \pm 6.31(a)$ & $41.29 \pm 7.85(a)$ & $43.71 \pm 8.50(a)$ & $45.57 \pm 10.47(a)$ & $49.14 \pm 13.47(a)$ \\
\hline & Megarhizo & $35.86 \pm 6.15(a)$ & $40.43 \pm 8.24(a)$ & $47.71 \pm 11,66(a)$ & $50.71 \pm 11.34(a)$ & $53.86 \pm 12.70(a)$ & $57.57 \pm 14.71(\mathrm{a})$ & $60.86 \pm 16.01(a)$ \\
\hline & Beyonik & $30.57 \pm 4.19$ (a) & $36.14 \pm 3.72(a)$ & $38.71 \pm 5.41(\mathrm{a})$ & $43.57 \pm 5.80(a)$ & $46.71 \pm 5.74(a)$ & $49.71 \pm 7.67(a)$ & $53.43 \pm 9.95(\mathrm{a})$ \\
\hline \multirow{3}{*}{5} & Control & $44.86 \pm 8.51(a)$ & $51.00 \pm 10.10(\mathrm{a})$ & $57.43 \pm 11.04(\mathrm{a})$ & $62.57 \pm 13.14(a)$ & $67.14 \pm 15.03(a)$ & $67.29 \pm 15.05(\mathrm{a})$ & $68.29 \pm 15.05(a)$ \\
\hline & Megarhizo & $51.71 \pm 7.99(\mathrm{a})$ & $59.57 \pm 8.50(a)$ & $65.86 \pm 9.79(a)$ & $72.00 \pm 11.06(\mathrm{a})$ & $76.71 \pm 14.36(\mathrm{a})$ & $78.57 \pm 14.32(a)$ & $79.43 \pm 14.27$ (a) \\
\hline & Beyonik & $43.86 \pm 13.93(a)$ & $51.86 \pm 17.77(a)$ & $59.00 \pm 21.38(\mathrm{a})$ & $67.43 \pm 23.70$ (a) & $70.00 \pm 25.85(a)$ & $71.86 \pm 26.37(a)$ & $72.57 \pm 26.54(\mathrm{a})$ \\
\hline \multirow{3}{*}{10} & Control & $26.83 \pm 4.54(a)$ & $30.67 \pm 3.83(a)$ & $33.67 \pm 4.80(a)$ & $38.00 \pm 5.18(a)$ & $44.00 \pm 7.48(a)$ & $44.33 \pm 7.74(a)$ & $45.17 \pm 7.65(\mathrm{a})$ \\
\hline & Megarhizo & $29.43 \pm 5.19$ (a) & $32.43 \pm 5.91(a)$ & $36.14 \pm 7.43(a)$ & $39.00 \pm 7.28(a)$ & $45.14 \pm 10.61(a)$ & $45.71 \pm 10.58(a)$ & $46.43 \pm 10.28(a)$ \\
\hline & Beyonik & $27.71 \pm 5.74(a)$ & $32.86 \pm 5.64(a)$ & $37.29 \pm 6.82(a)$ & $40.29 \pm 8.34(a)$ & $46.71 \pm 7.48(\mathrm{a})$ & $46.86 \pm 7.36(a)$ & $47.71 \pm 7.39(\mathrm{a})$ \\
\hline \multirow{3}{*}{13} & Control & $28.83 \pm 5.78(a)$ & $31.33 \pm 6.19$ (a) & $34.00 \pm 5.90(a)$ & $37.17 \pm 6.31(\mathrm{a})$ & $40.00 \pm 8.15(a)$ & $40.50 \pm 7.97(a)$ & $41.5 \pm 7.97(a)$ \\
\hline & Megarhizo & $32.43 \pm 6.16(a)$ & $37.43 \pm 6.95(a)$ & $44.29 \pm 9.18(b)$ & $48.29 \pm 8.32(b)$ & $53.57 \pm 11.86(b)$ & $54.00 \pm 11.40(\mathrm{~b})$ & $54.71 \pm 11.32(b)$ \\
\hline & Beyonik & $31.14 \pm 5.43(\mathrm{a})$ & $36.43 \pm 6.11(a)$ & $40.64 \pm 5.85(\mathrm{ab})$ & $44.86 \pm 6.82(\mathrm{ab})$ & $49.29 \pm 8.94(\mathrm{ab})$ & $49.57 \pm 8.98(a b)$ & $50.43 \pm 8.81(\mathrm{ab})$ \\
\hline \multirow{3}{*}{14} & Control & $44.00 \pm 3.00(a)$ & $46.86 \pm 6.07(a)$ & $54.00 \pm 7.40(a)$ & $60.14 \pm 9.48(a)$ & $63.14 \pm 8.43(\mathrm{a})$ & $65.00 \pm 6.40(a)$ & $68.86 \pm 8.45(a)$ \\
\hline & Megarhizo & $42.43 \pm 7.11(\mathrm{a})$ & $50.71 \pm 8.99$ (a) & $55.21 \pm 9.29$ (a) & $59.71 \pm 8.94(a)$ & $65.57 \pm 9.50(a)$ & $65.86 \pm 9.21(\mathrm{a})$ & $66.86 \pm 9.21(\mathrm{a})$ \\
\hline & Beyonik & $45.29 \pm 8.98(a)$ & $50.29 \pm 10.56(\mathrm{a})$ & $56.00 \pm 12.56(\mathrm{a})$ & $61.86 \pm 13.64(a)$ & $67.57 \pm 16.51(a)$ & $67.71 \pm 16.31(\mathrm{a})$ & $68.71 \pm 16.31(a)$ \\
\hline \multirow{3}{*}{28} & Control & $21.00 \pm 3.06$ (a) & $24.29 \pm 3.20$ (a) & $28.14 \pm 4.67(a)$ & $31.14 \pm 4.60(a)$ & $32.57 \pm 3.51(\mathrm{a})$ & $32.86 \pm 3.34(a)$ & $33.71 \pm 3.15(\mathrm{a})$ \\
\hline & Megarhizo & $20.29 \pm 2.75(a)$ & $24.57 \pm 4.54(a)$ & $27.29 \pm 5.44(a)$ & $32.43 \pm 4.76(a)$ & $34.43 \pm 5.38(a)$ & $34.71 \pm 5.28(a)$ & $35.43 \pm 5.26(\mathrm{a})$ \\
\hline & Beyonik & $20.00 \pm 2.89$ (a) & $23.29 \pm 4.61(a)$ & $28.00 \pm 6.08(a)$ & $31.00 \pm 4.62(\mathrm{a})$ & $34.29 \pm 5.19$ (a) & $34.29 \pm 5.19(\mathrm{a})$ & $35.14 \pm 5.27$ (a) \\
\hline
\end{tabular}

Values represents means \pm SE. Different letters in the same parameter data indicate significant differences by DUNCAN test (p $\leq 0.05$ ) 
TABLE VIII

GROWTH RATE (MM/MONTH) OF TRUNK DIAMETER OF GRAFTED AVOCADO

\begin{tabular}{|c|c|c|c|c|c|c|c|c|}
\hline \multirow{2}{*}{ Acc. } & \multirow{2}{*}{ Treatment } & \multicolumn{7}{|c|}{ Months After Bio-fertilizer Application } \\
\hline & & 5 & 7 & 9 & 11 & 13 & 15 & 17 \\
\hline \multirow{3}{*}{1} & Control & $0.167 \pm 0.142$ (a) & $0.170 \pm 0.138(a)$ & $0.076 \pm 0.133(a)$ & $0.146 \pm 0.728$ (a) & $0.056 \pm 0.032(\mathrm{a})$ & $0.000 \pm 0.000$ (a) & $0.011 \pm 0.007$ (a) \\
\hline & Megarhizo & $0.141 \pm 0.031(\mathrm{a})$ & $0.149 \pm 0.034(\mathrm{a})$ & $0.107 \pm 0.066$ (a) & $0.089 \pm 0.389$ (a) & $0.077 \pm 0.056$ (a) & $0.000 \pm 0.000$ (a) & $0.023 \pm 0.026$ (a) \\
\hline & Beyonik & $0.140 \pm 0.065(\mathrm{a})$ & $0.123 \pm 0.042(\mathrm{a})$ & $0.137 \pm 0.061(\mathrm{a})$ & $0.135 \pm 0.046(\mathrm{a})$ & $0.053 \pm 0.012(\mathrm{a})$ & $0.003 \pm 0.005(\mathrm{a})$ & $0.012 \pm 0.004(\mathrm{a})$ \\
\hline \multirow{3}{*}{2} & Control & $0.144 \pm 0.058$ (a) & $0.096 \pm 0.045(\mathrm{a})$ & $0.094 \pm 0.082$ (a) & $0.044 \pm 0.097(\mathrm{a})$ & $0.056 \pm 0.035(\mathrm{a})$ & $0.033 \pm 0.033(\mathrm{a})$ & $0.061 \pm 0.072(a)$ \\
\hline & Megarhizo & $0.189 \pm 0.061$ (a) & $0.107 \pm 0.067(\mathrm{a})$ & $0.146 \pm 0.049$ (a) & $0.061 \pm 0.030(\mathrm{a})$ & $0.057 \pm 0.043(\mathrm{a})$ & $0.063 \pm 0.049$ (a) & $0.053 \pm 0.035(\mathrm{a})$ \\
\hline & Beyonik & $0.177 \pm 0.038(\mathrm{a})$ & $0.157 \pm 0.052(\mathrm{a})$ & $0.140 \pm 0.175$ (a) & $0.109 \pm 0.082(\mathrm{a})$ & $0.069 \pm 0.050(\mathrm{a})$ & $0.056 \pm 0.044(\mathrm{a})$ & $0.063 \pm 0.039(\mathrm{a})$ \\
\hline \multirow{3}{*}{5} & Control & $0.171 \pm 0.056(\mathrm{a})$ & $0.119 \pm 0.043(\mathrm{a})$ & $0.111 \pm 0.047(a)$ & $0.077 \pm 0.049$ (a) & $0.063 \pm 0.049$ (a) & $0.001 \pm 0.004(\mathrm{a})$ & $0.014 \pm 0.005(\mathrm{a})$ \\
\hline & Megarhizo & $0.204 \pm 0.053$ (a) & $0.136 \pm 0.030$ (a) & $0.096 \pm 0.036$ (a) & $0.083 \pm 0.040(\mathrm{a})$ & $0.056 \pm 0.054(\mathrm{a})$ & $0.021 \pm 0.052$ (a) & $0.010 \pm 0.006(a)$ \\
\hline & Beyonik & $0.200 \pm 0.062$ (a) & $0.144 \pm 0.047$ (a) & $0.116 \pm 0.056$ (a) & $0.124 \pm 0.073(\mathrm{a})$ & $0.033 \pm 0.035(\mathrm{a})$ & $0.026 \pm 0.044(\mathrm{a})$ & $0.011 \pm 0.011(\mathrm{a})$ \\
\hline \multirow{3}{*}{10} & Control & $0.203 \pm 0.075$ (a) & $0.128 \pm 0.053$ (a) & $0.087 \pm 0.035$ (a) & $0.110 \pm 0.088$ (a) & $0.132 \pm 0.049$ (a) & $0.007 \pm 0.010$ (a) & $0.020 \pm 0.011(\mathrm{a})$ \\
\hline & Megarhizo & $0.253 \pm 0.072$ (a) & $0.089 \pm 0.073(\mathrm{a})$ & $0.100 \pm 0.037$ (a) & $0.076 \pm 0.052$ (a) & $0.130 \pm 0.047$ (a) & $0.013 \pm 0.013(\mathrm{a})$ & $0.016 \pm 0.011(\mathrm{a})$ \\
\hline & Beyonik & $0.204 \pm 0.051$ (a) & $0.161 \pm 0.041(\mathrm{~b})$ & $0.117 \pm 0.024$ (a) & $0.071 \pm 0.046$ (a) & $0.143 \pm 0.039$ (a) & $0.003 \pm 0.008$ (a) & $0.017 \pm 0.008$ (a) \\
\hline \multirow{3}{*}{13} & Control & $0.187 \pm 0.089$ (a) & $0.078 \pm 0.050$ (a) & $0.077 \pm 0.083(\mathrm{a})$ & $0.085 \pm 0.057$ (a) & $0.065 \pm 0.045(\mathrm{a})$ & $0.012 \pm 0.013(\mathrm{a})$ & $0.025 \pm 0.005(\mathrm{a})$ \\
\hline & Megarhizo & $0.204 \pm 0.060$ (a) & $0.134 \pm 0.057(\mathrm{~b})$ & $0.149 \pm 0.076(a)$ & $0.086 \pm 0.048(\mathrm{a})$ & $0.086 \pm 0.103$ (a) & $0.010 \pm 0.013(\mathrm{a})$ & $0.016 \pm 0.110$ (a) \\
\hline & Beyonik & $0.149 \pm 0.039$ (a) & $0.146 \pm 0.024(\mathrm{~b})$ & $0.106 \pm 0.034$ (a) & $0.090 \pm 0.077(\mathrm{a})$ & $0.083 \pm 0.062(\mathrm{a})$ & $0.006 \pm 0.010(\mathrm{a})$ & $0.019 \pm 0.009$ (a) \\
\hline \multirow{3}{*}{14} & Control & $0.140 \pm 0.084(\mathrm{a})$ & $0.051 \pm 0.103(\mathrm{a})$ & $0.127 \pm 0.089$ (a) & $0.099 \pm 0.055(\mathrm{a})$ & $0.050 \pm 0.057(\mathrm{a})$ & $0.030 \pm 0.067(\mathrm{a})$ & $0.051 \pm 0.092(\mathrm{a})$ \\
\hline & Megarhizo & $0.169 \pm 0.046$ (a) & $0.160 \pm 0.060(\mathrm{~b})$ & $0.081 \pm 0.042(a)$ & $0.077 \pm 0.052$ (a) & $0.090 \pm 0.042$ (a) & $0.006 \pm 0.010$ (a) & $0.017 \pm 0.005$ (a) \\
\hline & Beyonik & $0.183 \pm 0.029$ (a) & $0.097 \pm 0.043(\mathrm{ab})$ & $0.096 \pm 0.055$ (a) & $0.097 \pm 0.029$ (a) & $0.077 \pm 0.034(\mathrm{a})$ & $0.003 \pm 0.008(a)$ & $0.014 \pm 0.005(\mathrm{a})$ \\
\hline \multirow{3}{*}{28} & Control & $0.257 \pm 0.076(\mathrm{a})$ & $0.136 \pm 0.063(\mathrm{a})$ & $0.131 \pm 0.058(\mathrm{a})$ & $0.099 \pm 0.054(\mathrm{a})$ & $0.047 \pm 0.048(\mathrm{a})$ & $0.009 \pm 0.015(\mathrm{a})$ & $0.026 \pm 0.011(\mathrm{a})$ \\
\hline & Megarhizo & $0.304 \pm 0.073$ (a) & $0.164 \pm 0.100$ (a) & $0.096 \pm 0.037(\mathrm{a})$ & $0.159 \pm 0.100$ (a) & $0.057 \pm 0.048(\mathrm{a})$ & $0.009 \pm 0.015(\mathrm{a})$ & $0.020 \pm 0.014(\mathrm{a})$ \\
\hline & Beyonik & $0.251 \pm 0.075$ (a) & $0.134 \pm 0.069$ (a) & $0.163 \pm 0.083$ (a) & $0.100 \pm 0.098$ (a) & $0.094 \pm 0.081(\mathrm{a})$ & $0.000 \pm 0.000(\mathrm{a})$ & $0.024 \pm 0.011(\mathrm{a})$ \\
\hline
\end{tabular}

Values represents means \pm SE. Different letters in the same parameter data indicate significant differences by DUNCAN test (p $\leq 0.05$ )

TABLE IX

GROWTH RATE (MM/MONTH) OF TRUNK DIAMETER BASED ON BIO-FERTILIZER AND ACCESSION NUMBER

\begin{tabular}{|l|c|c|c|c|c|c|c|c|c|}
\hline \multirow{2}{*}{ Treatment } & \multicolumn{9}{|c|}{ Accession No. } \\
\cline { 2 - 11 } & 1 & 2 & 5 & 10 & 13 & 14 & 28 & Average & Rank \\
\hline Control & 0.112 & 0.09 & 0.092 & 0.117 & 0.089 & 0.09 & 0.122 & 0.102 & 3 \\
\hline Megarhizo & 0.096 & 0.111 & 0.101 & 0.118 & 0.116 & 0.098 & 0.15 & 0.113 & 1 \\
\hline Beyonic & 0.1 & 0.115 & 0.115 & 0.12 & 0.098 & 0.095 & 0.134 & 0.111 & 2 \\
\hline Average & 0.103 & 0.105 & 0.103 & 0.119 & 0.101 & 0.094 & 0.135 & & \\
\hline Rank & 5 & 3 & 4 & 2 & 6 & 7 & 1 & & \\
\hline
\end{tabular}

